\title{
Internal kinematics of spiral galaxies in distant rich galaxy clusters
}

\author{
K. Jäger ${ }^{1}$, A. Böhm ${ }^{1}$, B. L. Ziegler ${ }^{1}$, J. Heidt $^{2}$ and C. Möllenhoff ${ }^{2}$ \\ ${ }^{1}$ Universitäts-Sternwarte Göttingen, Germany, email: jaeger@uni-sw.gwdg.de \\ ${ }^{2}$ Landessternwarte Heidelberg, Germany
}

\begin{abstract}
We present our project on galaxy evolution in the environment of distant rich clusters aiming at disentangling the importance of specific interaction and galaxy transformation processes from the hierarchical evolution of field galaxies. Spatially resolved MOS spectra were gained with VLT/FORS to analyze the internal kinematics of disk galaxies. First results are shown for the clusters MS 1008.1-1224 $(z=0.30)$, Cl 0303+1706 $(z=0.42)$, and Cl 0413-6559 $(z=0.51)$. Out of 35 late-type cluster members, 13 galaxies exhibit a rotation curve of the universal form rising in the inner region and passing over into a flat part. The other members have peculiar kinematics. The 13 cluster galaxies for which a maximum rotation velocity could be derived are distributed in the Tully-Fisher diagram very similarly to field galaxies from the FORS Deep Field with corresponding redshifts. The same is true for seven non-cluster galaxies observed in the cluster fields. The TF-cluster spirals do not show any significant luminosity evolution as might be expected from certain phenomena specific to clusters. Contrary to that, the disturbed kinematics of the non-TF cluster spirals indicate ongoing or recent interaction processes.
\end{abstract}

\section{Introduction}

Galaxies in clusters reside in a special environment which is characterized by high galaxy densities, the presence of a hot intracluster medium (ICM) and vast amounts of dark matter. This environment strongly influences the evolution of the cluster members superposed on the field galaxy evolution that arises from the hierarchical growth of objects and the declining star formation rates over cosmic epochs. Besides tidal interactions (including merging) as known from the field, cluster galaxies are affected by phenomena, specific to clusters, related to the structure of the cluster (like harassment) or to the ICM (like ram-pressure stripping). These mechanisms can cause substantial distortions of the internal kinematics of disk galaxies leading to "rotation curves" (RCs) that no longer follow the universal form (Persic et al. 1996). Rubin et al. (1999), for example, classified half of their sample galaxies observed in the Virgo cluster as kinematically disturbed (asymmetric or even truncated curves). On the other hand, many cluster galaxies follow a tight Tully-Fisher relation (TFR) (e.g. Giovanelli et al. 1997).

An important idea presently discussed came from the finding that local clusters are dominated by elliptical and lenticular galaxies while the distant ones show a high fraction of spiral and irregular galaxies (Dressler et al. 1997): field spirals might fall into clusters and experience a morphological transformation into S0 galaxies. Related to this scenario it is not yet clear whether the halo of dark matter and, therefore, the total mass and the $\mathrm{RC}$ of a galaxy can also be affected by certain interaction phenomena.

First results on the kinematics of galaxies in distant clusters were presented by Metevier (2004) with $10 \mathrm{TF}$-galaxies in the cluster $\mathrm{Cl} 0024+1654(z=0.40)$ and by MilvangJensen et al. (2003) with 8 spirals in MS 1054.4-0321 ( $z=0.83)$. While the first study sees a larger scatter of their sample galaxies in the TF diagram without evidence for an 


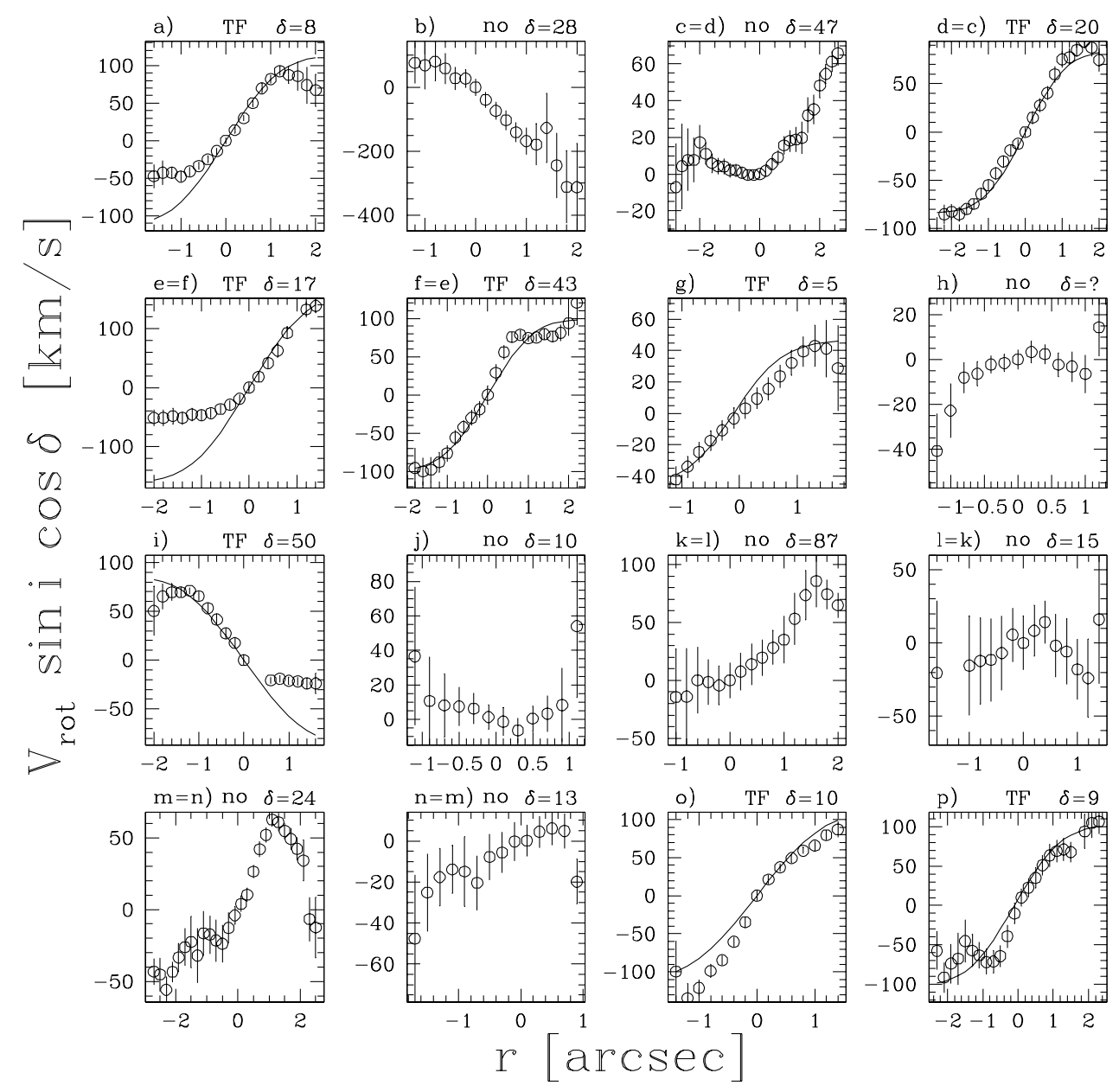

Figure 1. Spatially resolved velocity profiles for 12 disk galaxies in the cluster MS 1008-12 at $z=0.30$. Note, panels $c \& d$, e\&f, $k \& l$ and $m \& n$ represent the same galaxy, rsp., but observed with different slit positions (the rotation angles of the two MOS masks differed by $67^{\circ}$ ). $\delta$ gives the angle between slit direction and apparent major axis. Panels a-p are ordered according to the distance of the galaxies from the cluster center (upper left: smallest proj. radius). Values of the maximum rotation velocity could be determined for cases a, d, e, f, g, i, o \& p indicated by the label "TF". These galaxies enter the TFR shown in Fig. 2. In all other cases, the kinematics are too disturbed. In one case $(\mathrm{m} \& \mathrm{n})$, we observe a prominent bar that causes a highly asymmetric rotation curve.

evolution of the zero point, the second one finds a trend towards brighter luminosities (0.5-1 $B$ mag) with respect to field spirals of comparable redshifts. It is argued that either truncation of star formation or starbursts may cause an increased or decreased mass-to-light ratio, respectively.

\section{Project and observations}

During 5 nights at the ESO-VLT we gained spectra of galaxies within 7 clusters in the redshift range $0.3 \leqslant z<0.6$ with FORS $1 \& 2$. One of our goals was the derivation of spatially resolved RCs to analyse the internal kinematics of the galaxies and to construct 


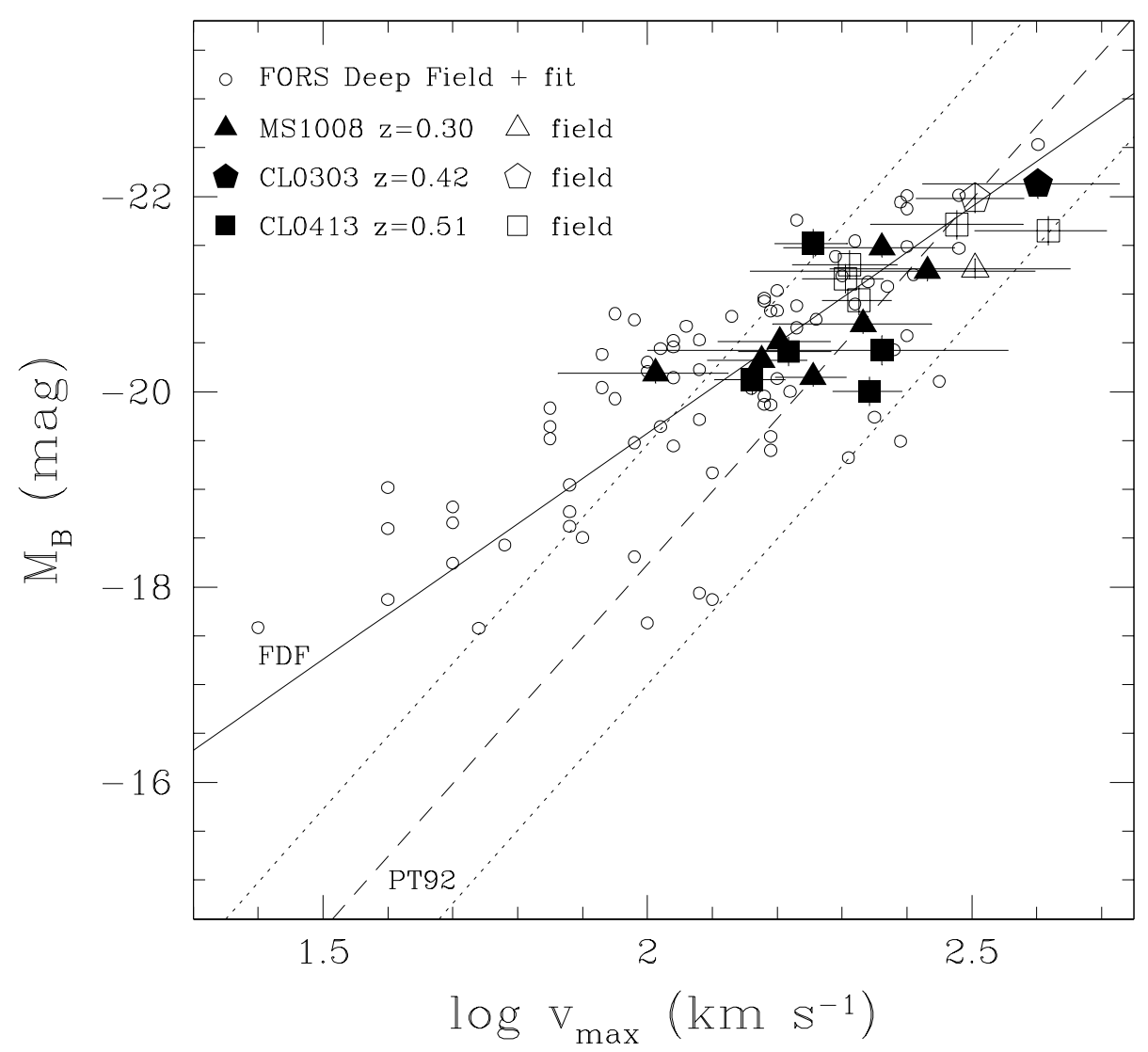

Figure 2. Tully-Fisher diagram of cluster spirals in MS 1008-12 $(z=0.30)$, Cl 0413-65 $(z=0.51)$ and $\mathrm{Cl} 0303+17(z=0.42)$. Also shown are 7 field objects (open symbols) that were also observed in the clusters' field-of-view. In comparison to our FORS Deep Field sample of 77 field galaxies with a mean redshift of 0.5 (small open circles), the cluster galaxies are similarly distributed and do not deviate significantly from the linear fit to the FDF sample (solid line). The cluster members follow the same trend with respect to the local TFR (the fit $\pm 3 \sigma$ to the Pierce \& Tully 1992 sample is given) as the distant field galaxies. Since we observed mostly bright cluster galaxies, their luminosities are not significantly increased in accordance with an undisturbed evolution. (Restframe $B$ magnitudes were calculated for a flat $\Omega_{\lambda}=0.7$ cosmology with $H_{0}=70 \mathrm{~km} \mathrm{~s}^{-1} \mathrm{Mpc}^{-1}$.)

the TFR (Tully and Fisher 1977) of distant clusters. HST/WFPC2 images of the core regions were retrieved from the archive to complement our own ground-based imaging. Here we present results of three of the clusters, Cl 0413-65 $(z=0.51)$ and MS 1008-12 $(z=0.30), \mathrm{Cl} 0303+17(z=0.42)$. Observations were made in 1999 and 2000 with FORS mounted at the Cassegrain focus of the VLT. We used grism 600R and get a spectral resolution of $R \approx 1200$ at a slitwidth of 1 arcsec. The spatial scale was $0.2 \mathrm{arcsec} / \mathrm{pixel}$ with a dispersion of $\sim 1.08 \AA /$ pixel. The total integration time was set to $\approx 2 \mathrm{hrs}$ to meet our signal-to-noise requirements. Seeing conditions ranged between 0.4 and 1.3 arcsec FWHM. Two MOS setups have been observed for each cluster, yielding 116 objects with apparent magnitudes in the range $18.0<R<23$. 


\section{Data reduction and rotation curve modelling}

A detailed description of the sample selection and data reduction including finding charts and data tables can be found in Jäger et al. (2004). For the derivation of position velocity diagrams we followed the way described in detail in Böhm et al. (2004) and Ziegler et al. (2003). Here we only give some short remarks on this topic.

All RCs were determined using either the $[\mathrm{O}$ II] $3727, \mathrm{H} \beta$ or $[\mathrm{O}$ III] 5007 emission line. Gaussian fits have been applied stepping along the spatial axis with a median filter window of typically 0.6 arcsecs to enhance the S/N. Since the apparent disk sizes of spirals at intermediate redshifts are only slightly larger than the slit width ( 1 arcsec), the spectroscopy covers a substantial fraction of the two-dimensional velocity field. Thus, the maximum rotation velocity $v_{\max }$ (the constant rotation in the outer part of a galaxy due to the Dark Matter halo) cannot be determined "straightforwardly" from the observed rotation. To tackle this problem, we simulated the spectroscopy of each galaxies' velocity field with the respective inclination and position angle, also taking seeing and luminosity weighting into account. The simulated rotation curves which best reproduced the observed ones (solid lines in Fig. 1) yielded the values of $v_{\max }$.

\section{Results and discussion}

Redshifts and spectral types could be determined for 89 galaxies. From the 50 cluster members, 35 turned out to be late type galaxies. Only 13 cluster galaxies exhibit a rotation curve of the universal form rising in the inner region and passing over into a flat part. The other members have peculiar kinematics or too low S/N. For 7 non-cluster galaxies $v_{\max }$ could also be measured.

Only those rotation curves which show no strong perturbations are eligible for a determination of $v_{\max }$ as needed for the TF diagram which is shown in Fig. 2. $v_{\max }$ could be measured for 7(MS 1008-12), 5(Cl0413-65), and 2(Cl0303+17) cluster members, respectively. Luminosities were derived from total magnitudes (measured with SExtractor, see Bertin \& Arnouts 1996) of $V$ (MS 1008-12), I (Cl0413-65), and $R$ $(\mathrm{Cl} 0303+17)$ FORS images corrected for Galactic and intrinsic extinction, transformed to restframe Johnson $B$ according to SED type, and calculated for a flat $\Omega_{\lambda}=0.7$ cosmology $\left(H_{0}=70 \mathrm{~km} \mathrm{~s}^{-1} \mathrm{Mpc}^{-1}\right)$.

Within our TF diagram the distant cluster spirals are distributed similar to the FORS Deep Field (FDF) spirals (Ziegler et al. 2002, Böhm et al. 2004) which have been observed with exactly the same instrument configuration and which represent a similar cosmic epoch $\left(\left\langle z_{\mathrm{FDF}}\right\rangle=0.5\right)$. No significant deviation from the distant field TFR is visible and the cluster sample has not an increased scatter, but the low number of cluster members prohibits any quantitative statistical analysis. We can conclude that the massto-light ratios of the observed distant cluster spirals cover the same range as the distant field population indicating that no phenomenon specific to clusters dramatically changed the stellar populations. In particular, there was no starburst in the recent past of the examined cluster galaxies that would have significantly risen their luminosities. Since we mostly selected bright galaxies, the cluster members occupy a region in the TF diagram where no significant luminosity evolution is visible with respect to the local TFR.

On the other hand, this conclusion is true only for those objects that enter the TF diagram and is not valid for the whole cluster sample. More than half of our cluster galaxies can not be used for a TF analysis due to their peculiar kinematics not following the "rise-turnover-flat" RC shape of large, isolated spirals. This is in contrast to the smaller fraction of peculiar curves in our sample of the FDF spirals. The objects 
with peculiar RCs may actually be subject to ongoing or recent interactions. Indeed, a morphological classification of the cluster spirals in terms of their asymmetry indices (Abraham et al. 1996) reveals that they have a slightly higher degree of asymmetry on the mean than the kinematically less disturbed ones. A correlation between morphological and kinematical disturbance hints to a common origin for both in "strong" interactions like close encounters with tidal effects, accretion events or even mergers. Such processes most probably also influence the stellar populations of a galaxy changing its integrated luminosity as well.

No trend is visible of the $\mathrm{RC}$ form with (projected) clustercentric distance (cf. Fig.1). But since all observed galaxies are located within the virial radius, this is in compliance with dynamical models in which the galaxy population of a cluster is well-mixed within that region. In particular, we most probably do not have any new arrivals from the field in our sample.

\section{Acknowledgements}

Based on observations with the ESO-VLT (64.O-0158 \& 64.O-0152). This work has been supported by the Volkswagen Foundation (I/76 520) and the Deutsche Forschungsgemeinschaft (Fr 325/46-1 and SFB 439).

\section{References}

Abraham et al. 1996 MNRAS 279, L47.

Bertin E., \& Arnouts, S. 1996 A $\& A S$ 117,393.

Böhm, A., Ziegler, B. L., Saglia, R.P., et al. $2004 A \mathscr{E} A$ in press (astro-ph/0309263)

Dressler, A. et al. $1997 \mathrm{ApJ}$ 490, 577

Giovanelli, R., Haynes, M. P., Herter, et al. 1997 AJ 113, 22

Jäger, K., Ziegler, B.L., Böhm, A., et al. $2004 A \xi A$ submitted

Kodama, T., \& Bower, R. G. 2001 MNRAS 321, 18

Metevier, A. J. 2004. In Clusters of Galaxies: Probes of Cosmological Structure and Galaxy Evolution, eds. Mulchaey, J.S, Dressler, A., Oemler, A. Carnegie Obs. Astrophysics Series Vol.3

Milvang-Jensen, B., Aragón-Salamanca, A., Hau, G. K. T., et al. 2003 MNRAS 339, L1

Persic, M., Salucci, P., \& Stel, F. 1996 MNRAS 281, 27

Pierce, M. J. \& Tully, R. B. 1992 ApJ 387, 47

Rubin, V. C., Waterman, A. H., Kenney, J. D. P. 1999 AJ 118, 236

Tully, R.B., \& Fisher, J.R. 1977 A $\mathscr{S} A$ 54, 661

Ziegler, B.L., Böhm, A., Fricke, K.J., et al. 2002 ApJL 564, L69

Ziegler, B.L., Böhm, A., Jäger, K., et al. 2003 ApJL 598, L87 